\title{
A Rare Case of Ascariasis-Induced Cholangitis Complicated With Klebsiella pneumoniae Bacteremia and Liver Microabscesses
}

\author{
Mohammad Aldiabat $^{1}$, Yasir Saeed ${ }^{1}$, Donya Bani Hani ${ }^{1}$, Sami Rabah ${ }^{1}$, Bo Yu ${ }^{1}$ \\ 1. Internal Medicine, Lincoln Medical Center, New York, USA
}

Corresponding author: Mohammad Aldiabat, mohameddiabat88@gmail.com

\begin{abstract}
Ascariasis, which is caused by Ascaris lumbricoides, is the most common gastrointestinal parasitic infection worldwide, with occasional invasion of the biliary tract leading to a variety of complications. In rare cases, pathogens carried on the surface of $A$. lumbricoides can complicate the course of the disease and lead to superimposed bacterial infections. In this article, we present a case of ascariasis-induced cholangitis complicated with Klebsiella pneumoniae bacteremia and multiple hepatic microabscesses. This article, which shows an association that was not reported in the literature before, aims to increase the awareness of clinicians for the possibility of the association between ascariasis and superimposed bacterial infection, specifically with $K$. pneumoniae.
\end{abstract}

Review began $12 / 26 / 2020$ Review ended 12/30/2020 Published 01/05/2021

(c) Copyright 2021

Aldiabat et al. This is an open access article distributed under the terms of the Creative Commons Attribution License CC-BY 4.0., which permits unrestricted use, distribution, and reproduction in any medium, provided the original author and source are credited.
Categories: Internal Medicine, Gastroenterology, Infectious Disease

Keywords: cholangitis, microabscess, liver, bacteremia, klebsiella pneumoniae (kp), ascaris lumbricoides

\section{Introduction}

Ascariasis, which is caused by Ascaris lumbricoides, is the most common gastrointestinal parasitic infection worldwide [1], with occasional invasion of the biliary tract leading to a variety of complications including cholecystitis, pyogenic cholangitis, and liver abscess. In rare cases, pathogens carried on the surface of $A$. lumbricoides can complicate the course of the disease and lead to superimposed bacterial infections. In this article, we present a case of a 17-year-old male who presented with ascariasis-induced cholangitis complicated with Klebsiella pneumoniae bacteremia and multiple hepatic microabscesses. To our knowledge, this association has not been reported in the literature before. The authors of this article aim to increase the awareness of clinicians for the possibility of the association between ascariasis and superimposed bacterial infection, specifically with $K$. pneumoniae.

\section{Case Presentation}

We present a case of a 17-year-old Hispanic male with a past medical history of Roux-en-y choledochojejunostomy, who presented to the hospital with a five-day history of fever, non-radiating right upper quadrant (RUQ) abdominal pain, nausea, vomiting, diarrhea, generalized weakness, confusion, and loss of appetite. The patient had watery, non-bilious, non-bloody vomiting and diarrhea, with no obvious foreign body passage. There was no history of skin discoloration or pruritus. The patient moved to the United States from Dominican Republic two years ago. Physical exam was remarkable for a young afebrile male with diaphoresis, restlessness, confusion, and RUQ tenderness. No abdominal distention or hepatomegaly was noted. Murphy, McBurney, and Rovsing signs were negative. The laboratory findings are reviewed in Table 1. 


\section{Cureus}

\begin{tabular}{|c|c|c|c|}
\hline Test & First admission & Second admission & Reference \\
\hline WBC & 4.5 & 19.95 & $4.80-10.80 \times 10^{3} / \mathrm{mcL}$ \\
\hline Eosinophils count & 0.1 & 0.04 & $0.10-0.40 \times 10^{3} / \mathrm{mcL}$ \\
\hline Eosinophils (\%) & 1.4 & 0.2 & $1.0-4.0$ \\
\hline AST (SGOT) & 23 & 84 & $\leq 40 \mathrm{U} / \mathrm{L}$ \\
\hline ALT (SGPT) & 25 & 59 & $\leq 41 \mathrm{U} / \mathrm{L}$ \\
\hline ALK PHOS & 103 & 125 & $40-130 \mathrm{U} / \mathrm{L}$ \\
\hline Total bilirubin & 1.24 & 2.12 & $0.20-1.20 \mathrm{mg} / \mathrm{dL}$ \\
\hline Direct bilirubin & 0.4 & 1 & $0.00-0.30 \mathrm{mg} / \mathrm{dL}$ \\
\hline Albumin & 4.1 & 4 & $3.5-5.2 \mathrm{~g} / \mathrm{dL}$ \\
\hline Lipase, serum & 19 & 12 & $13-60 \mathrm{U} / \mathrm{L}$ \\
\hline Amylase, serum & 62 & 55 & $40-130 \mathrm{U} / \mathrm{L}$ \\
\hline Procalcitonin & 4.46 & 20.46 & $\leq 0.08 \mathrm{ng} / \mathrm{mL}$ \\
\hline Blood cultures & Klebsiella pneumoniae & Klebsiella pneumoniae & Sterile \\
\hline Hepatitis B surface antigen & Nonreactive & Nonreactive & Nonreactive \\
\hline Hepatitis C antibodies & Nonreactive & Nonreactive & Nonreactive \\
\hline Stool ova and parasite & Negative & Negative & Negative \\
\hline Malaria and parasite screen & Negative & Negative & Negative \\
\hline Blood parasites & Negative & Negative & Negative \\
\hline Strongyloides antibodies & Negative & Negative & Negative \\
\hline Clostridium difficile toxins $\mathrm{A} / \mathrm{B}$ antigen, stool & Negative & Negative & Negative \\
\hline Giardia antigen & Not detected & Not detected & Not detected \\
\hline Entamoeba histolytica serolog & Negative & Negativ & Negative \\
\hline
\end{tabular}

\section{TABLE 1: Laboratory results.}

ALK PHOS, alkaline phosphatase; ALT, alanine aminotransferase; AST, aspartate aminotransferase; SGOT, serum glutamic oxaloacetic transaminase; SGPT, serum glutamic pyruvic transaminase; WBC, white blood cells

Abdominal CT scan (Figure 1) with contrast showed multiple spheroidal foreign bodies within dilated biliary tracts, enhancement of segment VIII and VII suggestive of inflammation, regional adenopathy, and pneumobilia within the biliary tree of the left lobe due to previous choledochojejunostomy. Magnetic resonance cholangiopancreatography (MRCP) (Figure 2) confirmed previous findings of intrahepatic biliary dilatation in the right lobe of liver with multiple curvilinear filling defects within the ducts with a $15-\mathrm{mm}$ conglomeration centrally within the posterior segment of the right lobe, suspicious for parasitic infestation. Given the patient demographics and clinical and imaging findings, the patient was diagnosed with biliary parasitic infection (likely biliary ascariasis being the most common etiology) complicated with acute cholangitis and severe sepsis. The patient was admitted to pediatric intensive care unit for close monitoring and was started on broad-spectrum antibiotics. Stool testing was negative for ova and parasite, which can be explained by the absence of eggs secondary to an infection with a male $A$. lumbricoides. Blood cultures showed growth of pan-sensitive K. pneumoniae; therefore, antibiotics were adjusted to ceftriaxone IV, and the patient received praziquantel and albendazole to cover ascariasis and other parasitic infections. Following administration of anti-parasitic agents, the patient's clinical status improved significantly over his five-day stay, with resolution of symptoms. Repeated blood culture showed resolution of bacteremia. The patient was discharged with a seven-day course of cefdinir and nitazoxanide. 


\section{Cureus}

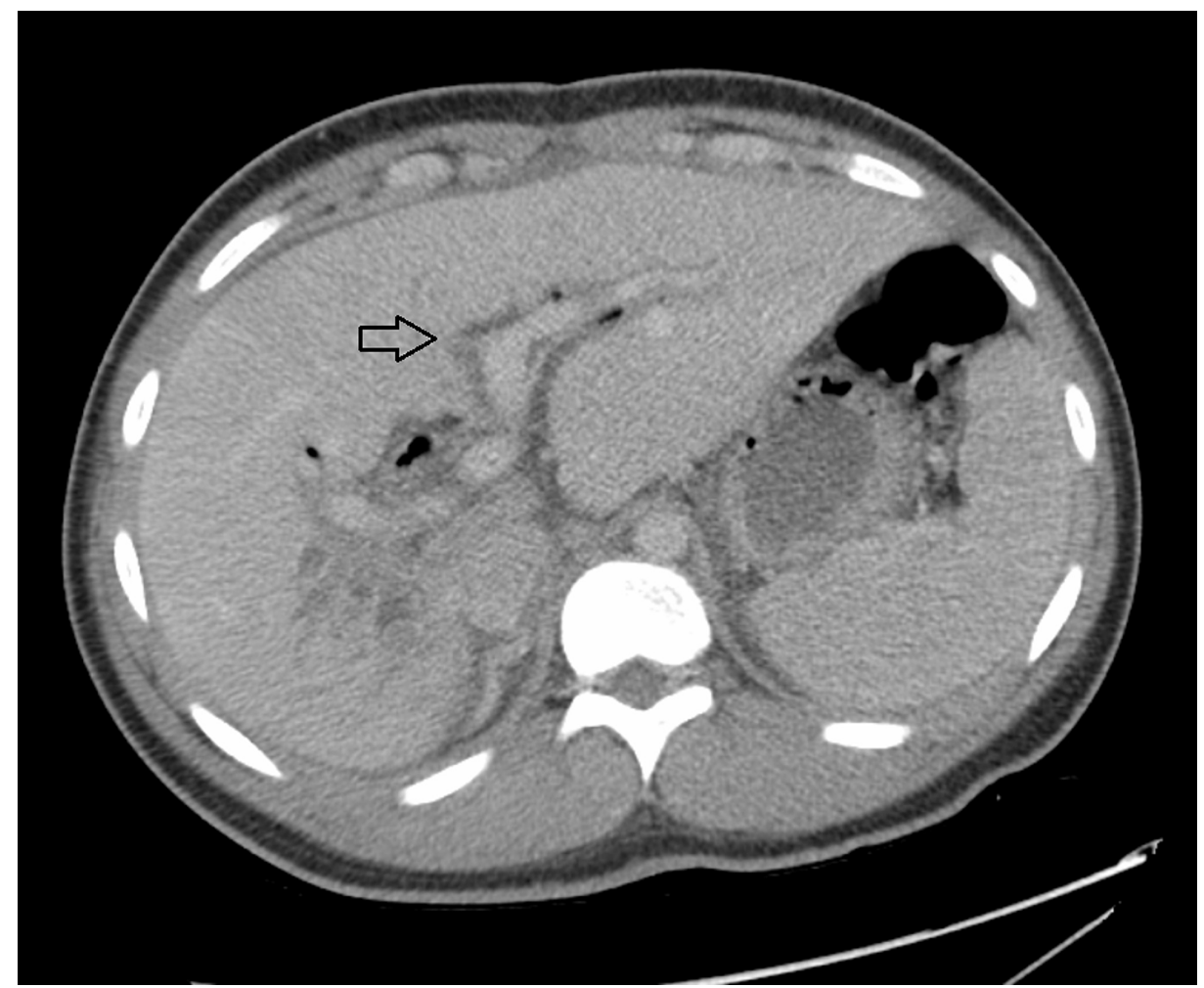

FIGURE 1: Abdominal CT scan showing multiple spheroidal foreign bodies within the dilated biliary tracts.

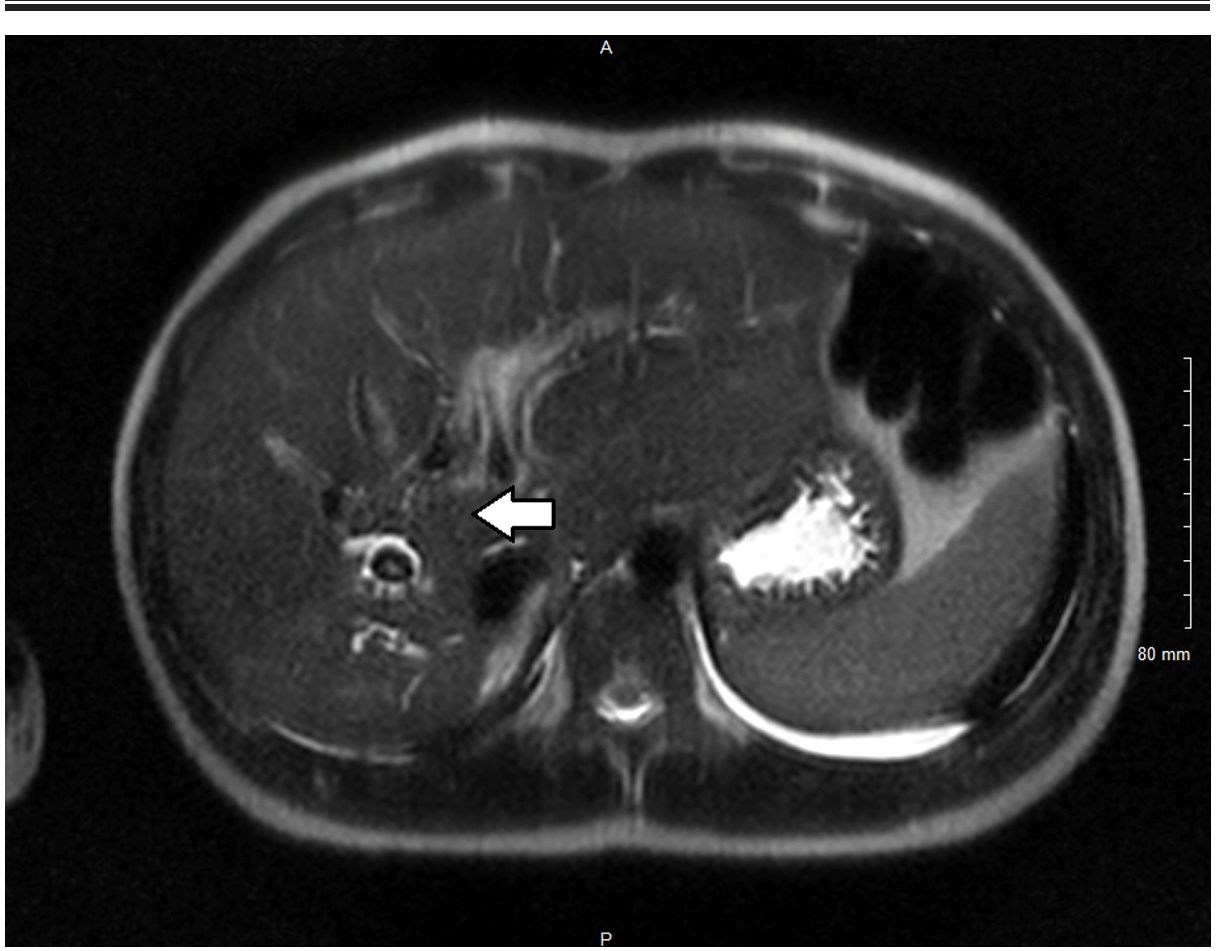

FIGURE 2: Magnetic resonance cholangiopancreatography confirmed findings of intrahepatic biliary dilatation in the right lobe of the liver with multiple curvilinear filling defects.

Seven months later, the patient presented with similar complaints of fever, abdominal pain, vomiting, and diarrhea. Repeat laboratory testing is reviewed in Table 1. Blood cultures grew K. pneumoniae. Repeat CT 


\section{Cureus}

scan (Figure 3) showed innumerable poorly defined foci of diminished attenuation in the right lobe of the liver, representing multiple hepatic microabscesses (that were poorly defined on previous scans), with persistent intrahepatic biliary ducts dilation representing reinfection of A. lumbricoides. Ova and parasite screen in addition to Entamoeba histolytica serology were negative. The patient received albendazole $400 \mathrm{mg}$ orally, with clinical improvement and clearance of bacteremia after four days. On the seventh-week followup, repeat CT scan of the patient (Figure 4) showed significant improvement of the previous intrahepatic biliary dilatation and complete resolution of the hepatic microabscesses.

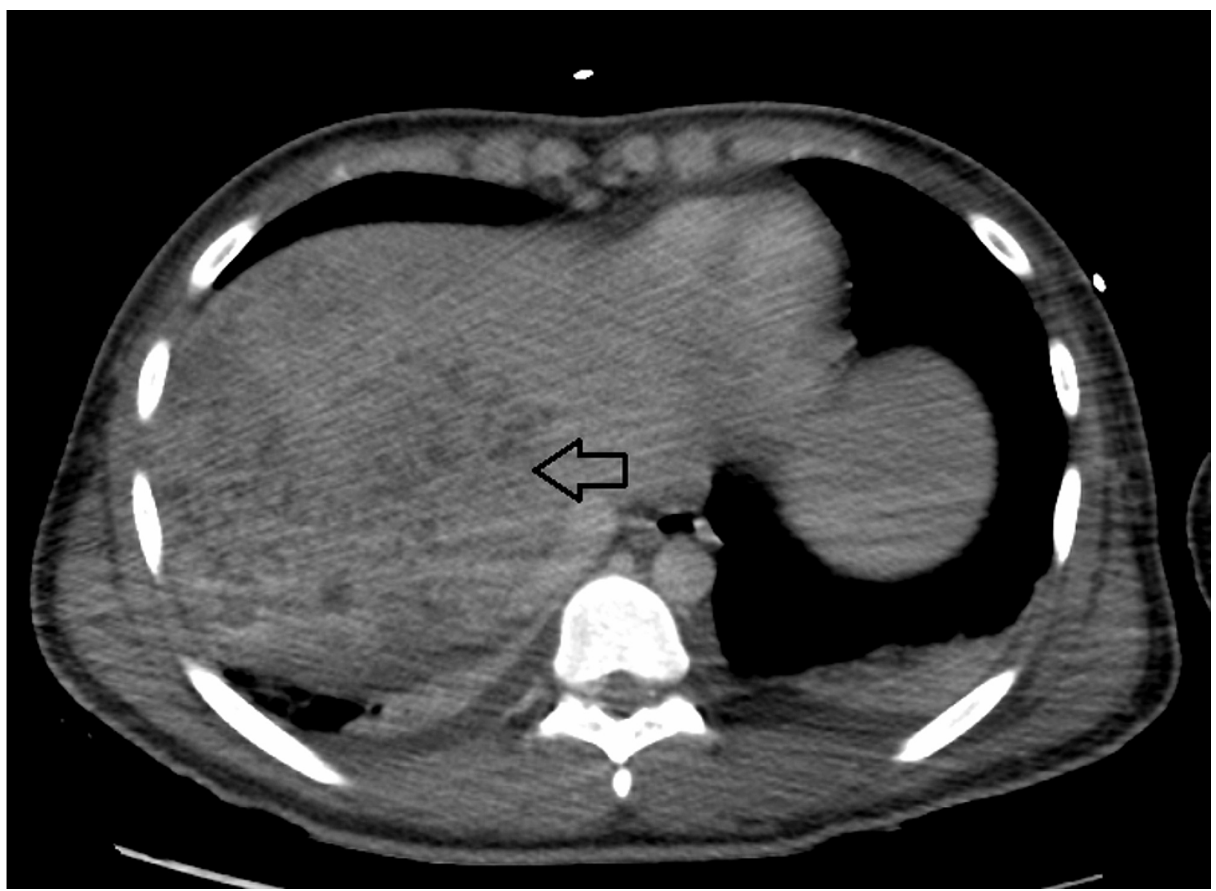

FIGURE 3: Repeat CT scan showed innumerable poorly defined foci of diminished attenuation in the right lobe of the liver representing multiple hepatic microabscesses.

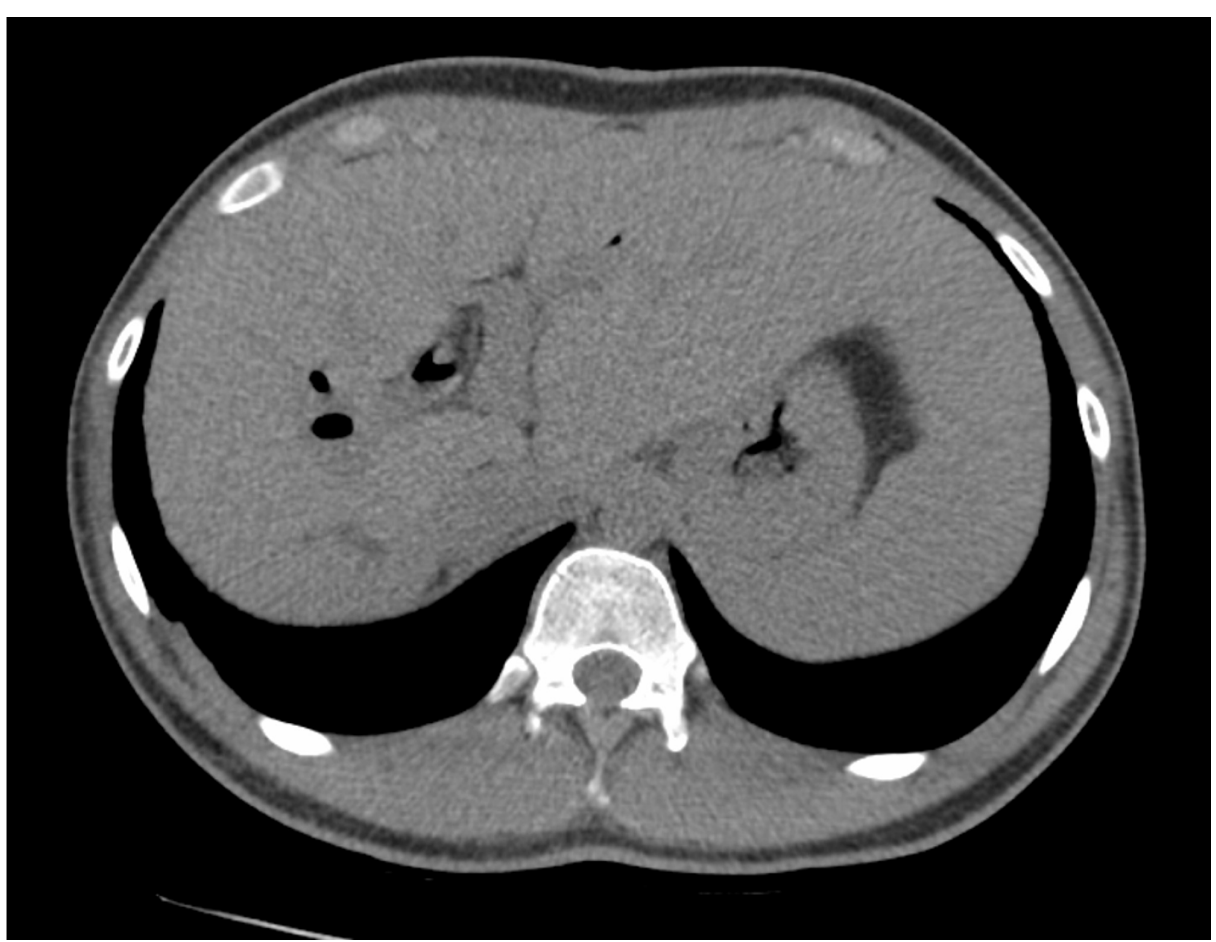

FIGURE 4: Significant improvement of intrahepatic biliary dilatation and 


\section{Discussion}

Ascariasis is the most common helminthic infection worldwide, with an estimated prevalence of 891 million around the world to be infected with the largest hookworm in the gastrointestinal tract [1]. The clinical scenario of intestinal ascariasis is usually benign, but in endemic areas such as India, it was found to be associated with one-third of patients with biliary and pancreatic diseases [2]. Ascaris lumbricoides can migrate to biliary duct through ampulla of Vater, where it can stay for up to three weeks and be complicated with biliary colic, biliary strictures, acalculous cholecystitis, ascending cholangitis, obstructive jaundice, and bile duct perforation with peritonitis before it migrates back again to the duodenum [3]. Rarely, penetration of liver parenchyma and colonization can happen in less than $1 \%$ of cases; thus, ascariasis-induced liver abscess should be suspected more in endemic areas of the world, such as Asia, Africa, and South America [4]. Treatment with benzimidazole (one dose of albendazole $400 \mathrm{mg}$ ) has a cure rate of $96.6 \%$ and an egg reduction rate of $99.9 \%$ [5], but reinfection can happen within six months secondary to persistent risk factors of poor sanitation and overcrowded houses [6], which may explain our patient's reinfection. In addition, anti-parasitic agents may not be fully effective against parasites present in the biliary tree, as these medications are poorly excreted in bile, and, therefore, surgical intervention may be required [7]. However, the patient presented in our case responded well to treatment with oral albendazole, and surgical intervention was not indicated.

Pyogenic liver abscess is a potential life-threatening situation, which is commonly caused by enteric Gramnegative bacilli such as K. pneumoniae and Escherichia coli [8]. Invasive liver abscess syndrome, an entity that describes liver abscess that occurs in the absence of hepatobiliary disease, is frequently reported in Asia (especially in Taiwan [9]), South Africa, and among Hispanic patients [10], hence our patient. In our case, the patient developed pyogenic cholangitis secondary to biliary ascariasis and was complicated with Klebsiella bacteremia and subsequent multiple hepatic microabscesses, an association, to our knowledge, that was not reported in the literature before. A retrospective study of 10 patients with ascariasis-induced liver abscess showed that blood cultures of all patients were sterile, whereas pus culture grew $E$. coli in four of them [11]. The presence of $A$. lumbricoides as a pathogen carrier of enteric $K$. pneumoniae explains the presence of bacteremia with the same pathogen during both admissions, which was complicated with multiple hepatic microabscesses on the second admission.

\section{Conclusions}

Ascariasis is the most common helminthic infection worldwide, with biliary tree involvement happening in up to one-third of cases in endemic areas. Rarely, ascaris can serve as a carrier for enteric flora on its surface, which leads to secondary hepatobiliary infection and liver abscess formation. Ascariasis associated with liver abscess is a rare finding, and, prior to this report, its association with Klebsiella bacteremia has not been reported in the literature.

\section{Additional Information \\ Disclosures}

Human subjects: Consent was obtained by all participants in this study. Conflicts of interest: In compliance with the ICMJE uniform disclosure form, all authors declare the following: Payment/services info: All authors have declared that no financial support was received from any organization for the submitted work. Financial relationships: All authors have declared that they have no financial relationships at present or within the previous three years with any organizations that might have an interest in the submitted work. Other relationships: All authors have declared that there are no other relationships or activities that could appear to have influenced the submitted work.

\section{References}

1. Pullan RL, Smith JL, Jasrasaria R, Brooker SJ: Global numbers of infection and disease burden of soil transmitted helminth infections in 2010. Parasit Vectors. 2014, 7:37. 10.1186/1756-3305-7-37

2. Javid G, Wani NA, Gulzar GM, Khan BA, Shah AH, Shah OJ, Khan M: Ascaris-induced liver abscess. World J Surg. 1999, 23:1191-4. 10.1007/s002689900645

3. de S Rocha M, Costa NS, Costa JC, et al.: CT identification of ascaris in the biliary tract . Abdom Imaging. 1995, 20:317-9. 10.1007/BF00203362

4. Sanai FM, Al-Karawi MA: Biliary ascariasis: report of a complicated case and literature review . Saudi J Gastroenterol. 2007, 13:25-32. 10.4103/1319-3767.30462

5. Samuel F, Degarege A, Erko B: Efficacy and side effects of albendazole currently in use against Ascaris, Trichuris and hookworm among school children in Wondo Genet, southern Ethiopia. Parasitol Int. 2014, 63:450-5. 10.1016/j.parint.2013.10.014

6. Wardell R, Clements ACA, Lal A, et al.: An environmental assessment and risk map of Ascaris lumbricoides and Necator americanus distributions in Manufahi District, Timor-Leste. PLoS Negl Trop Dis. 2017, 11:0005565. 10.1371/journal.pntd.0005565

7. Chakrabarti I, Giri A, De A, Roy AC: Radio-pathological diagnosis of hepatobiliary ascariasis: a rare entity . J 


\section{Cureus}

Cytol. 2011, 28:114-6. 10.4103/0970-9371.83468

8. Johannsen EC, Sifri CD, Madoff LC: Pyogenic liver abscesses. Infect Dis Clin North Am. 2000, 14:547-63. 10.1016/S0891-5520(05)70120-3

9. Yang CC, Yen $\mathrm{CH}$, Ho MW, Wang JH: Comparison of pyogenic liver abscess caused by non-Klebsiella pneumoniae and Klebsiella pneumoniae. J Microbiol Immunol Infect. 2004, 37:176-84.

10. Braiteh F, Golden MP: Cryptogenic invasive Klebsiella pneumoniae liver abscess syndrome. Int J Infect Dis. 2007, 11:16-22. 10.1016/j.ijid.2005.10.006

11. Bari S, Sheikh KA, Ashraf M, Hussain Z, Hamid A, Mufti GN: Ascaris liver abscess in children. J Gastroenterol. 2007, 42:236-40. 10.1007/s00535-006-1989-5 